\title{
MicroRNA-187 modulates epithelial-mesenchymal transition by targeting PTRF in non-small cell lung cancer
}

\author{
YANJUN CAI ${ }^{1,2^{*}}$, JIAN RUAN $^{3 *}$, XUEQING YAO $^{4}$, LIANG ZHAO $^{5}$ and BAOCHENG WANG ${ }^{1,6}$ \\ ${ }^{1}$ Department of Oncology, Jinan Clinical College of the Second Military Medical University, Jinan, Shandong; \\ ${ }^{2}$ Department of Geriatrics, General Hospital of Guangzhou Military Command of PLA, Guangzhou, Guangdong; \\ ${ }^{3}$ Cancer Center, Traditional Chinese Medicine-Integrated Hospital, Southern Medical University, Guangzhou, Guangdong; \\ ${ }^{4}$ Department of General Surgery, Guangdong General Hospital, Guangdong Academy of Medical Science, Guangzhou, \\ Guangdong; ${ }^{5}$ Department of Pathology, School of Basic Medical Sciences, Southern Medical University, Guangzhou, \\ Guangdong; ${ }^{6}$ Department of Oncology, General Hospital of Jinan Military Command of PLA, Jinan, Shandong, P.R. China
}

Received November 11, 2016; Accepted December 14, 2016

DOI: $10.3892 /$ or.2017.5548

\begin{abstract}
MicroRNAs (miRNAs) that negatively regulate gene expression play a key role in the development and progression of cancer. Aberrant expression of hsa-miR187 (miR-187) has been reported in various malignancies. However, the function of miR-187 in tumor progression remains controversial and its role in non-small cell lung cancer (NSCLC) is poorly understood. In the present study, the role of miR-187 in the progression of NSCLC was investigated. Our results revealed that miR-187 was frequently upregulated in NSCLC tissues and cells. Furthermore, ectopic introduction of miR-187 promoted cell migration, whereas miR-187 inhibitor had the contrary effect in NSCLC cells. Of significance, miR-187 induced epithelial-mesenchymal transition (EMT), which plays a pivotal role in the initiation of metastasis and activated mitogen-activated protein kinase (MAPK) and phosphatidylinositol-3-kinase/protein kinase B (PI3K/AKT) pathways. Polymerase I and transcript release factor (PTRF) was identified as a direct target of miR-187 in the promotion of the migration of NSCLC cells. Restored expression of PTRF neutralized the promoting effect of miR-187 on cell migration and EMT of NSCLC cells. Collectively, our data highlight the pivotal role of miR-187 in the progression of NSCLC, indicating this factor as a potential candidate in molecular cancer therapy.
\end{abstract}

Correspondence to: Dr Baocheng Wang, Department of Oncology, Jinan Clinical College of The Second Military Medical University, Jinan, Shandong, P.R. China

E-mail: baocheng-wang@hotmail.com

*Contributed equally

Key words: non-small cell lung cancer, epithelial-mesenchymal transition, polymerase I and transcript release factor, PTRF, microRNAs, tumor metastasis

\section{Introduction}

Lung cancer remains the leading cause of cancer-related deaths worldwide (1). Non-small cell lung cancer (NSCLC) accounts for $\sim 85 \%$ of all lung cancer subtypes and its clinical course is very crucial with poor patient outcomes $(2,3)$. Despite recent advances in chemotherapy and surgical procedures, the overall 5 -year survival rate of NSCLC patients $(\sim 15 \%)$ has not markedly improved (1). Adjacent invasion and distant metastases are responsible for the failure of lung cancer therapy (4). Therefore, further exploration of the underlying mechanisms of NSCLC progression is essential.

Epithelial-mesenchymal transition (EMT) is a key stage in tumor progression. This biological process permits polarized epithelial cells to assume a mesenchymal cell phenotype, however this often induces tumor metastasis (5). Increasing evidence supports EMT as a common event in human advanced NSCLC $(6,7)$. Consequently, to identify new diagnostic strategies and specifically targeted drugs, exploring key molecules in EMT that control NSCLC metastasis is paramount.

MicroRNAs (miRNAs), a family of small non-coding RNAs, are processed from precursor RNAs with a typical hairpin secondary structure (8). Several miRNAs are aberrantly expressed in NSCLC and their dysregulation has resulted in cancer progression and poor clinical outcome (9-11). Considering that miRNAs usually act as EMT-associated downstream effectors of receptor signaling or protein kinases (12), miRNAs may represent novel targets for designing antimetastatic drugs due to their specificity. Moreover, miRNA efficacy data is now available, making miRNA-based technology applicable against NSCLC metastasis in vivo (13).

Recently, miR-187 ectopic expression was detected in various types of cancer including nasopharyngeal (14), renal (15), pancreatic (16), prostate (17) and neuroblastoma (18). In NSCLC, miR-187 is also a potential tumor suppressor (19), however the precise molecular mechanism through which miR-187 influences NSCLC progression remains largely unknown. In the present study, we aimed to investigate the 
potential role of miR-187 in NSCLC progression. We also explored the mechanisms underlying tumor metastasis and direct targets of miR-187.

\section{Materials and methods}

Cell culture. Human NSCLC cell lines A549, H322, GLC-82, SPC-A1, PC-9, H460 and H1299, and the normal human bronchial epithelial cell line BEAS-2B, were obtained from the Cell Bank of the Chinese Academy of Sciences (Shanghai, China). All cells were authenticated by short tandem repeat (STR) profiling before receipt and were propagated for $<6$ months after resuscitation. All cells were cultured in RPMI-1640 medium (HyClone, Logan, UT, USA) supplemented with $10 \%$ fetal bovine serum (FBS; Gibco-BRL, Invitrogen, Paisley, UK) at room temperature with a humidity of $90-95 \%$, and $5 \% \mathrm{CO}_{2}$.

miRNA transfection. Refer to our previous study for details (20).

Microarray data set analysis. A microarray data set containing 116 paired primary lung cancers, and their corresponding adjacent normal lung tissues (collected a minimum of $5 \mathrm{~cm}$ from the tumor) were retrieved from the Gene Expression Omnibus (GEO) database (accession no. GSE15008) (21).

RNA isolation, reverse transcription and quantitative real-time polymerase chain reaction ( $q R T$-PCR). qRT-PCR for miRNAs and target genes was performed as previously described (20,22). Primer sequences used for PCR assays were as follows: U6 forward, 5'-CTCGCTTCGGCAGCAGCACA-3' and reverse, 5'-AACGCTTCACGAATTTGCGT-3'; and miR-187 forward, 5'-TCGTGTCTTGTGTTGCAGCC-3' and reverse, 5'-TGGTGTCGTGGAGTCG-3'.

Western blot analysis. Protein expression was evaluated by immunoblot analysis of cell lysates (20-60 $\mu \mathrm{g}$ ) in RIPA buffer solution using a rabbit antibody to E-cadherin, and mouse antibodies to $\beta$-catenin, fibronectin, vimentin and $\beta$-actin (1:500; Santa Cruz Biotechnology, Santa Cruz, CA, USA); rabbit antibodies to p-Akt (Ser473), AKT, p44/42 MAPK (ERK1/2), and p-p44/42 MAPK (ERK1/2) [1:1,000; Cell Signaling Technology (CST), Danvers, MA, USA]; and rabbit antibodies to polymerase I and transcript release factor (PTRF) (1:500; Abcam, Cambridge, UK).

Immunofluorescence (IF). Refer to our previous study for details (20). In brief, slides were incubated with a rabbit antibody to E-cadherin, mouse antibodies to $\beta$-catenin and vimentin (1:500; Santa Cruz Biotechnology), and rabbit antibody to PTRF (1:100; Abcam) at $4^{\circ} \mathrm{C}$ overnight followed by washing with phosphate-buffered saline (PBS) 3 times. Coverslips were then incubated with fluorescein isothiocyanate (FITC)- or Texas Red (TR)-conjugated anti-mouse or anti-rabbit secondary antibodies (1:120; Santa Cruz Biotechnology) for $30 \mathrm{~min}$ at room temperature prior to staining with 4'-6-diamidino-2-phenylindole (DAPI; Invitrogen, Carlsbad, CA, USA).

Cell migration analysis. Refer to our previous study for details (20).
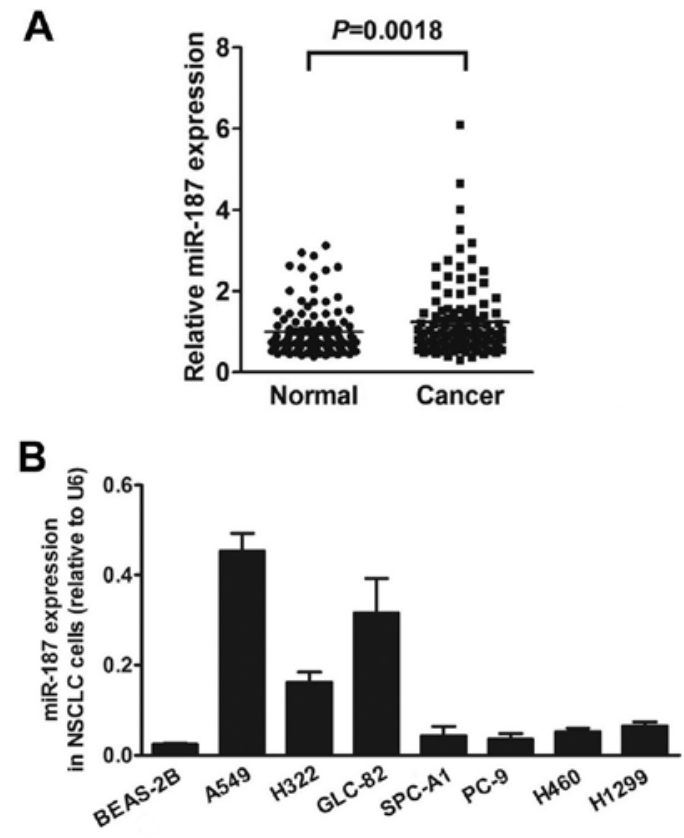

Figure 1. miR-187 is upregulated in NSCLC tissues and cell lines. (A) miR-187 expression in paired human NSCLC and their adjacent normal mucosal tissues from a published high-throughput microarray dataset (NCBI/GEO/ GSE15008; $n=116$ ). miR-187 expression was normalized to the matched adjacent normal tissues. (B) Increased expression of miR-187 was exhibited in all 7 NSCLC cell lines compared with the normal human bronchial epithelial cell line, BEAS-2B. Error bars represent the mean \pm SD from 3 independent experiments. NSCLC, non-small cell lung cancer.

Wound-healing assay. Refer to our previous study for details (20).

miRNA target validation. The full-length PTRF 3' untranslated regions (3'UTRs) were amplified by PCR and cloned downstream of the firefly luciferase gene in the psiCHECK-2 vector (Promega, Madison, WI, USA). This vector was named the wild-type (wt) 3'UTR. Site-directed mutagenesis of the miR-187 binding site in the PTRF 3'UTR was conducted with the GeneTailor Site-Directed Mutagenesis System (Invitrogen) and named the mutant $(\mathrm{mt}) 3^{\prime} \mathrm{UTR}$. For reporter assays, the wt or $\mathrm{mt}$ 3'UTR and miR-187 mimic or inhibitor were cotransfected. The luciferase activity was assessed 2 days after transfection using the Dual-Luciferase Reporter Assay System (Promega). Primer sequences used for PCR assays were as follows: WT PTRF forward, 5'-CCGCTCGAGGAGGCAGCCCCCG CAGTGGACAAC-3' and reverse, 5'-ATAAGAATGCGGCC GCGAAAAGCAGGTTTATTGGTCGGGC-3'; MutPTRF-1 forward, 5'-TGATTCTGTTTGGACTGGGTTCTCATCTGT GCTCCAGGTTCTCAAGACACGAGTCCCC-3' and reverse 5'-GGGGACTCGTGTCTTGAGAACCTGGAGCACAGAT GAGAACCCAGTCCAAACAGAATCA-3'; MutPTRF-2 forward, 5'-TTCTCAAGACACGACCAGGTTCTCATCTGT GCTGTCCCCTTGTTCCTCCCCATTAAAG-3' and reverse 5'-CTTTAATGGGGAGGAACAAGGGGACAGCACAGAT GAGAACCTGGTCGTGTCTTGAGAA-3'; and MutPTRF-3 forward, 5'-TTCTCATCTGTGCTCCAGGTTCTCATCTGT GCTGTCCCCTTGTTCCTCCCCATTAAAG-3' and reverse 5'-CTTTAATGGGGAGGAACAAGGGGACAGCACAGAT GAGAACCTGGAGCACAGATGAGAA-3'. 
A
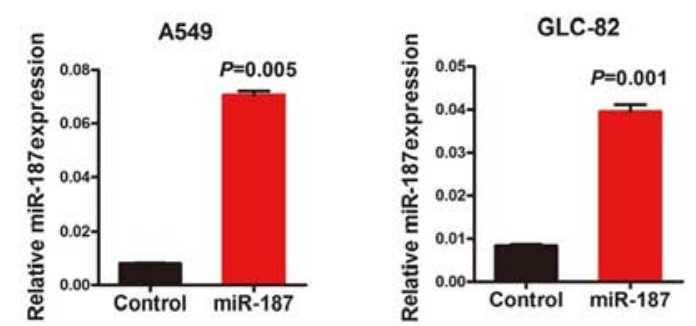

B
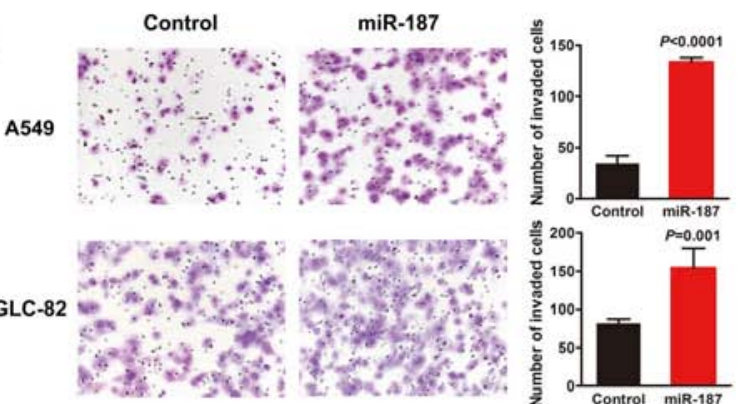

C
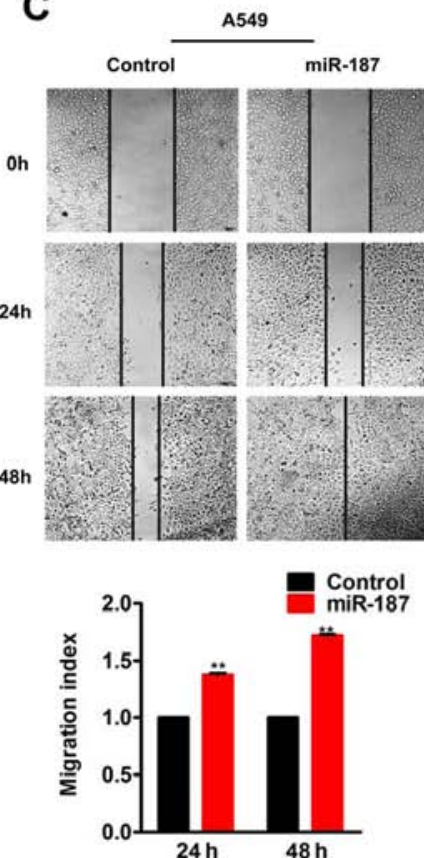
miR-187
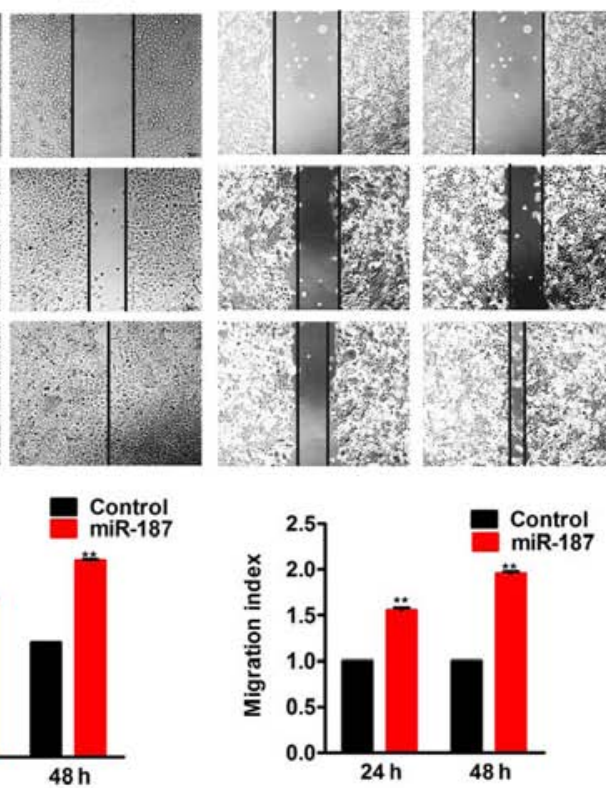

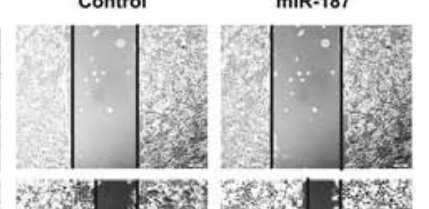

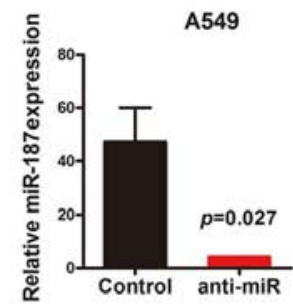
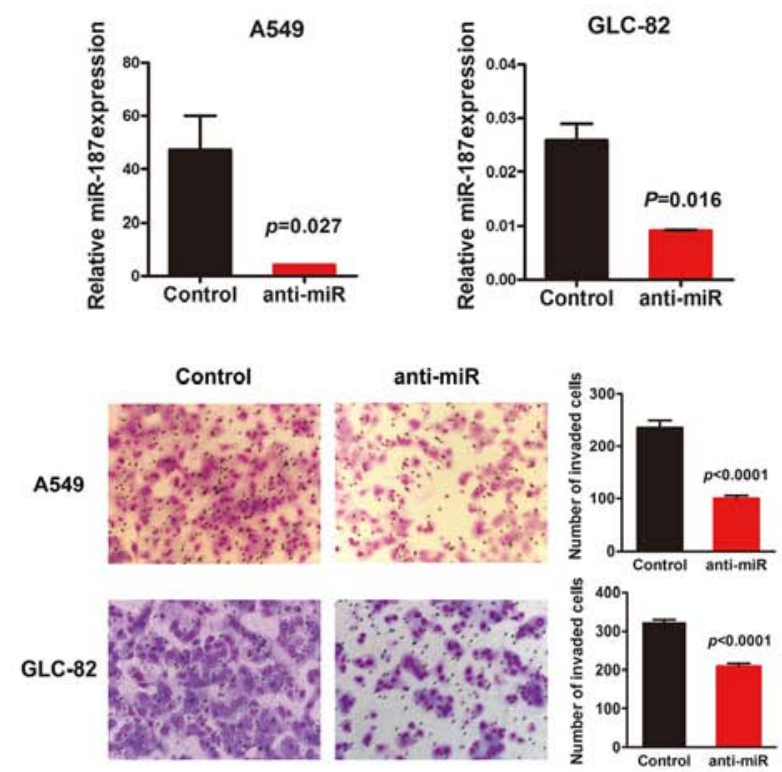
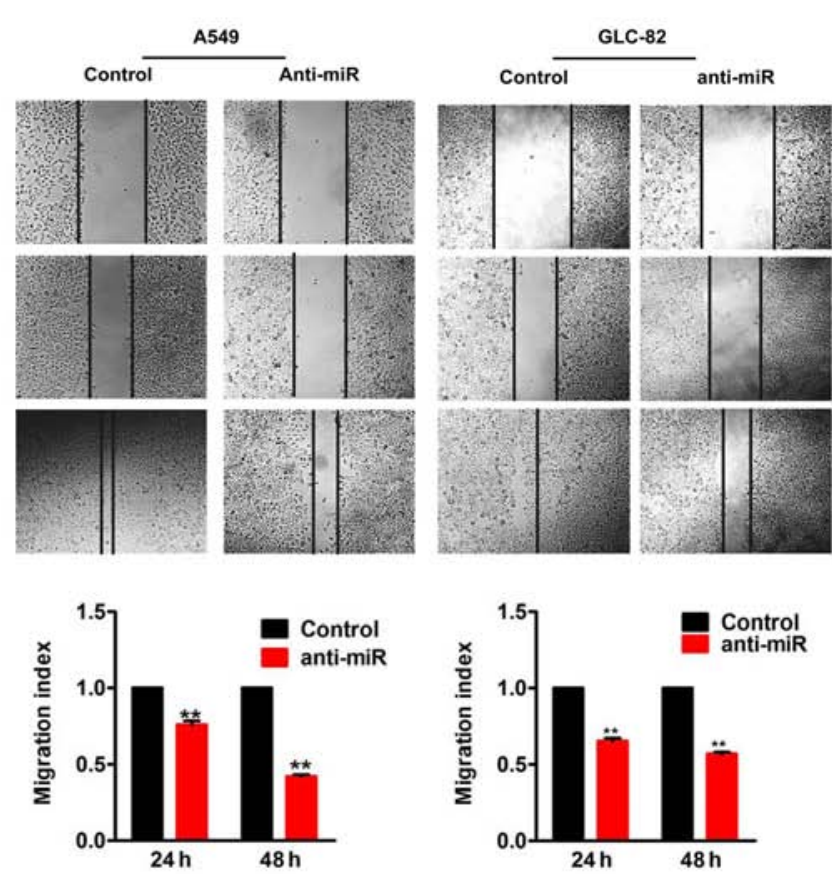

Figure 2. Exogenous miR-187 promotes NSCLC cell migration and motility in vitro. (A) RT-PCR analysis of miR-187 expression in miR-187 or anti-miR-187-transduced cells. Transcript levels were normalized to U6 expression. (B) Transwell assay using control and miR-187-overexpressing or -knockdown cell lines. The cells were counted under a microscope in 5 randomly selected fields. The histogram at the right shows the number of invaded cells. (C) Representative images of the wound-healing assay using control and miR-187-overexpressing or -knockdown cell lines. The lower histograms show the migration index of treated or control cells. The distance migrated by the treated cells was relative to that migrated by the control cells. Each bar represents the mean $\pm \mathrm{SD}$. The results were reproduced from 3 independent experiments; ${ }^{* *} \mathrm{P}<0.01$. NSCLC, non-small cell lung cancer.

Statistical analysis. Data were analyzed using SPSS software (version 19.0; SPSS, Inc., Chicago, IL, USA). For the qRT-PCR, Transwell and luciferase reporter assays, the Student's t-test or one-way analysis of variance (ANOVA) was carried out. All data are presented as the mean \pm standard deviation (SD). Statistical significance was established when the P-value was $<0.05$.

\section{Results}

miR-187 is upregulated in NSCLC tissues and cell lines. Microarray analysis showed that miR-187 was upregulated at an average of 1.26 -fold $(\mathrm{P}=0.0018)$ in the primary lung neoplasms compared with the corresponding adjacent normal lung tissues. Up to a 4.99-fold change in miR-187 expression was found in 71 of all 116 NSCLC samples compared to the controls (Fig. 1A). Furthermore, increased expression of miR-187 was found in all 7 NSCLC cell lines compared with the normal human bronchial epithelial cell line BEAS-2B (Fig. 1B).

Exogenous miR-187 promotes NSCLC cell migration and motility in vitro. In the present study, miR-187 mimic oligonucleotides were transfected into A549 and GLC-82 
A
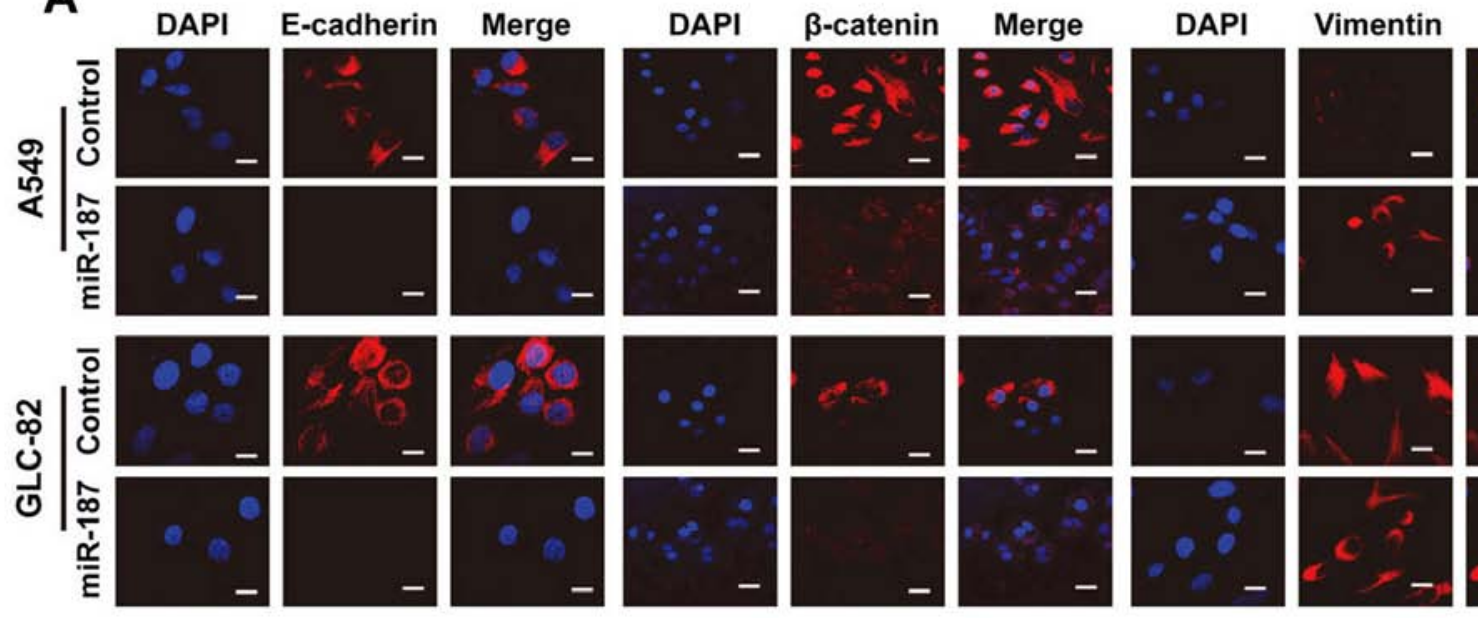

Merge
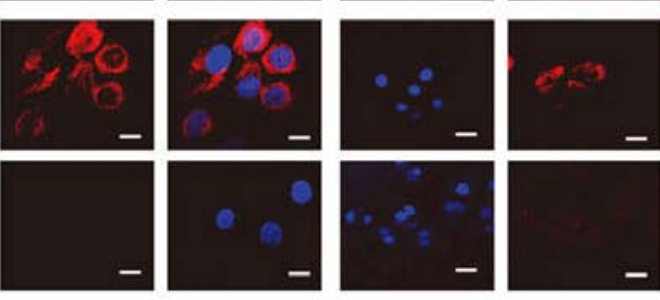

B

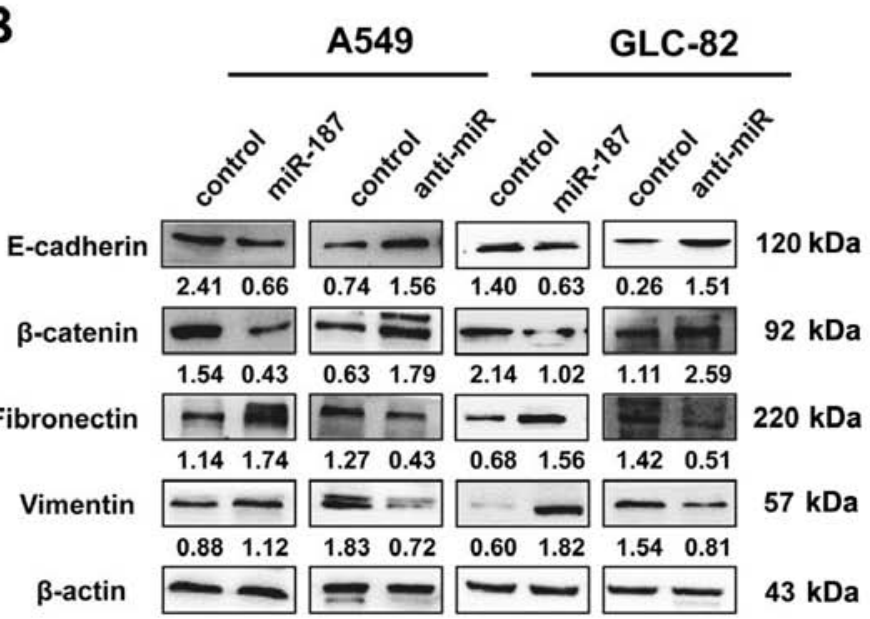

C
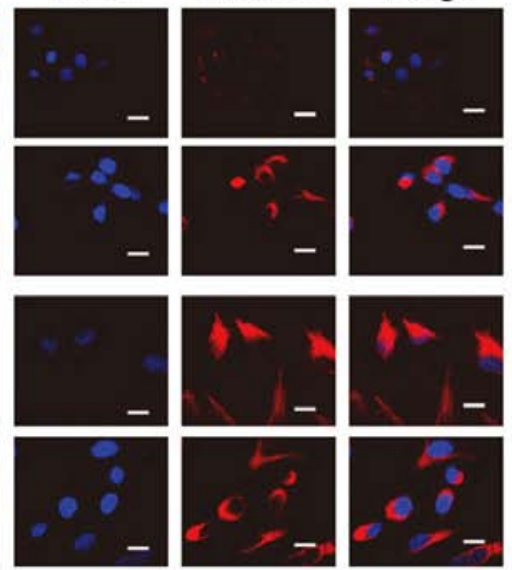

A549

GLC-82

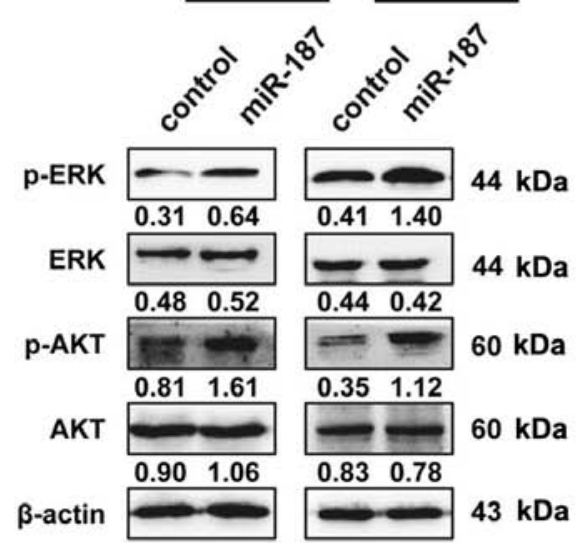

Figure 3. miR-187 mediates EMT and activates signal transduction pathways. (A) IF assays were performed to observe the localization of EMT markers (Scale bar, $10 \mu \mathrm{m}$ ). (B) Western blot analysis was performed to detect EMT markers. (C) Western blot analysis was performed to detect phosphorylation levels of ERK and AKT. The immunosignal was quantified using densitometric scanning software and the relative protein abundance was determined by normalization with $\beta$-actin. Representative figures are shown. The results were reproduced from 3 independent experiments. EMT, epithelial-mesenchymal transition; IF, immunofluorescence.

cell lines to determine their effects on cellular behavior. In contrast, anti-miR-187 was utilized to observe the effect of miR-187-depletion on cellular behavior as an miRNA inhibitor. qRT-PCR was performed to detect the transfection efficiency $(\mathrm{P}<0.05$; Fig. 2A). miR-187-treated cells showed a remarkable increase in migration and motility potential in Transwell and wound-healing assays, whereas depletion of endogenous miR-187 significantly decreased migration and motility $(\mathrm{P}<0.05$; Fig. 2B and $\mathrm{C})$.

miR-187 mediates EMT and activates signal transduction pathways. To investigate the mechanisms underlying miR-187-mediated biological behavior, we investigated its effects on EMT and signal transduction pathways. IF assays revealed that exogenous miR-187 overexpression resulted in decreased expression of the epithelial markers E-cadherin and $\beta$-catenin, and increased expression of the mesenchymal marker vimentin (Fig. 3A). Western blot analysis revealed similar changes in EMT markers (Fig. 3B) and indicated that the phosphorylation status of proteins was involved in EMT signaling. As shown in Fig. 3C, miR-187 activated the mitogen-activated protein kinase (MAPK) pathway through the phosphorylation of p44/42 MAPK (ERK1/2) and phosphatidylinositol 3-kinase/protein kinase B (PI3K/AKT) signaling via the phosphorylation of AKT in A549 and GLC-82 cells.

PTRF is a direct target of miR-187. As indicated by computer-based sequence analysis (based on TargetScan Human 6.2, PicTar and miRanda), PTRF was identified as a potential target of miR-187 and therefore considered to be involved in cell migration. The target sequence (wt 3'UTR) or 2 mutant sequences (mt 3'UTR position 1764-1790) were cloned into a luciferase reporter vector (Fig. 4A), then 293T cells were transfected with the wt or mt 3'UTR vector and an miR-187 mimic. A significant decrease in luciferase activity was observed in both the wt and mt vectors compared with the miR controls, whereas increased activity was found in cells cotransfected with anti-miR-187 and the wt or mt 3'UTR vectors (Fig 4B). Unlike for double mutations, single mutations in the putative binding sites in the PTRF 3'UTR region could not abrogate this suppression, thereby providing strong evidence of direct interaction between miR-187 and 
A
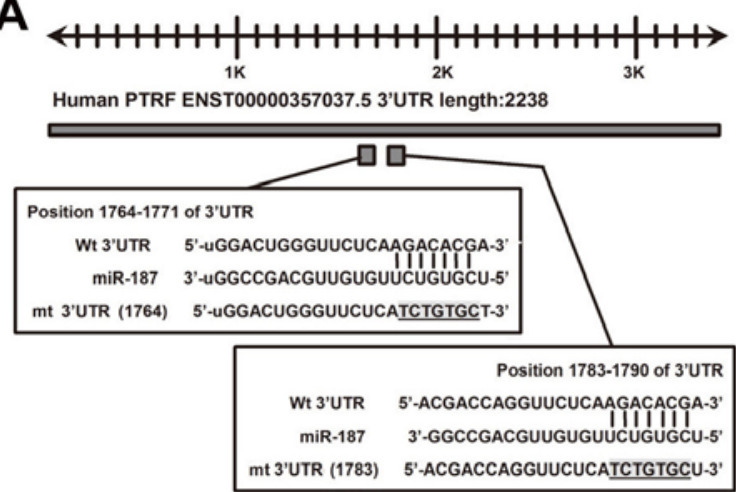

C
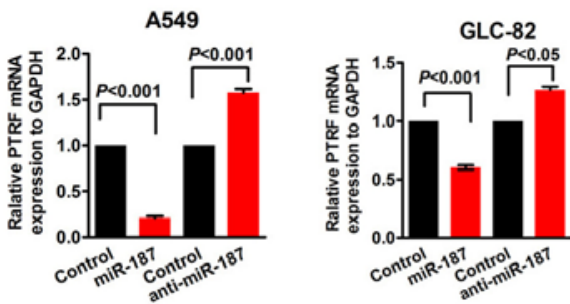

D

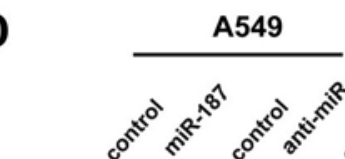

PTRF
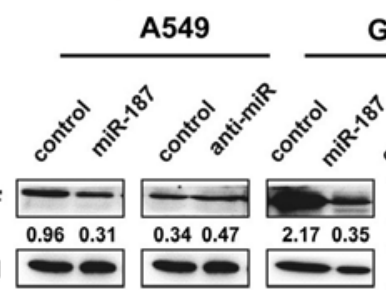

GLC-82

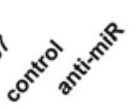

GAPDH

.

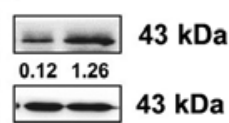

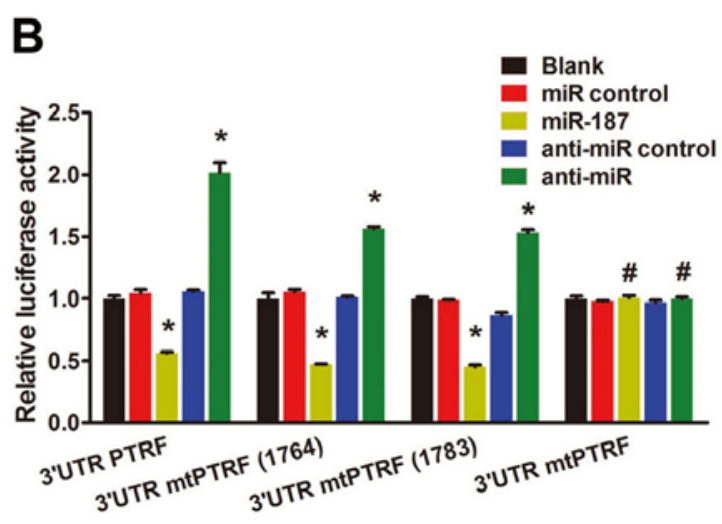

E

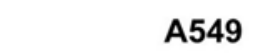

A549
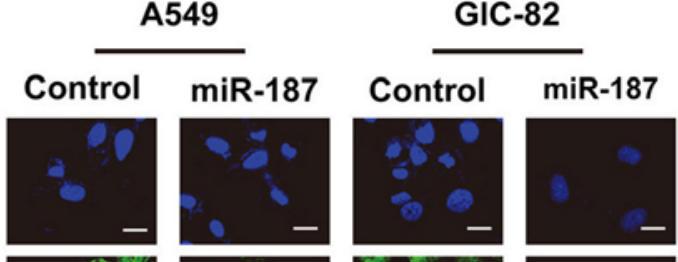

PTRF
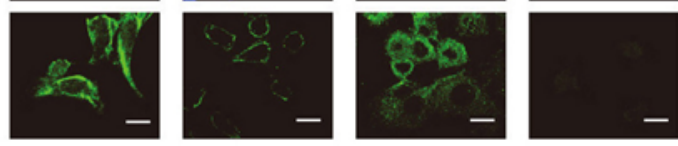

Merge
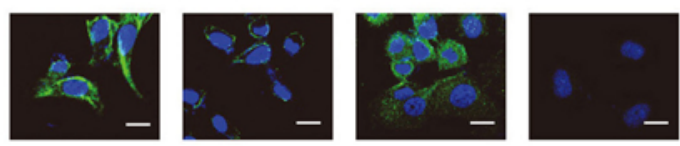

Figure 4. PTRF is a direct target of miR-187. (A) Diagram of PTRF 3'UTR containing 2 putative conserved target sites for miR-187, identified using the TargetScan database. (B) Results of luciferase reporter assays in 293T cells, with cotransfection of wt or mt 3'UTR and miR mimic and inhibitor, as indicated; ${ }^{*} \mathrm{P}<0.05$ and ${ }^{\#} \mathrm{P}>0.05$. (C) qRT-PCR analysis of PTRF mRNA proteins in indicated cells transfected with miR-187 mimic. Each bar represents the mean \pm SD. (D) Western blot analysis of PTRF protein expression in indicated cells after transfection with miR-187 mimic or inhibitor. Results were reproduced from 3 independent experiments. (E) IF assays were performed to observe localization of the PTRF protein (Scale bar, $10 \mu \mathrm{m}$ ). PTRF, polymerase I and transcript release factor; IF, immunofluorescence.

PTRF (Fig. 4B). In general, these results strongly suggest that PTRF is a direct target of miR-187 in NSCLC cells.

Additionally, qRT-PCR revealed decreased expression of PTRF in A549 and GLC-82 cells transfected with miR-187 (Fig. 4C). Western blot analysis revealed that transfection of miR-187 led to a markedly decreased expression of PTRF proteins in NSCLC cells (Fig. 4D), which were confirmed by IF assays to be potential target genes regulated by miR-187 (Fig. 4E).

PTRF plays crucial roles in miR-187-induced EMT and migration of NSCLC cells. To further explore the function and role of PTRF in NSCLC, we performed gain-of-function assays. Relatively decreased expression of PTRF was found in H460, GLC-82, SPC-A1 and A549 NSCLC cell lines compared with the normal human bronchial epithelial cell line BEAS-2B (Fig. 5A). Exogenous introduction of PTRF markedly enhanced PTRF protein expression (Fig. 5B) and decreased the capacity of cell migration in A549 and GLC-82 cells (Fig. 5C).

To evaluate the biological role of PTRF in cell migration and EMT induced by miR-187, we rescued PTRF expression in miR-187-transfected NSCLC cells by transfection with PTRF ORF constructs without 3'UTRs. The results revealed that exogenous introduction of PTRF clearly suppressed miR-187-induced promotion of migratory potential in A549 and GLC-82 cells (Fig. 6A). Consistent with the biological phenotype results, re-expression of PTRF recovered miR-187-induced EMT (Fig. 6B), strongly supporting PTRF as a key mediator of miR-187-induced cell migration.

\section{Discussion}

Our present results highlight the importance of upregulated miR-187 expression for NSCLC cells to develop and/or sustain their aggressive phenotype. Aberrant expression of miR-187 has been reported in various types of cancer (14-18). Although changes in expression vary greatly, miR-187 may participate in tumorigenesis in a tissue-specific manner. However, data on the effects of miR-187 in tumor progression are controversial. For example, in one study, antisense-induced suppression of miR-187 caused the inhibition of cell viability in HeLa cells (23), whereas overexpression of miR-187 induced apoptosis and led to decreased proliferation of $\mathrm{HeLa}$ cells in another study (24). Hence, enriching our knowledge regarding specific tumors may contribute to better clinical management. In NSCLC, miR-187 was found to be a potential tumor suppressor in tumor development (19), but the precise 
A

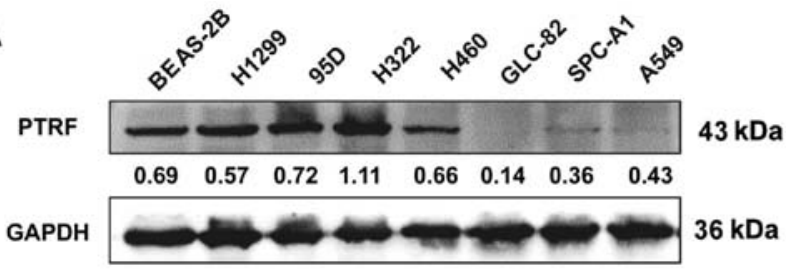

C
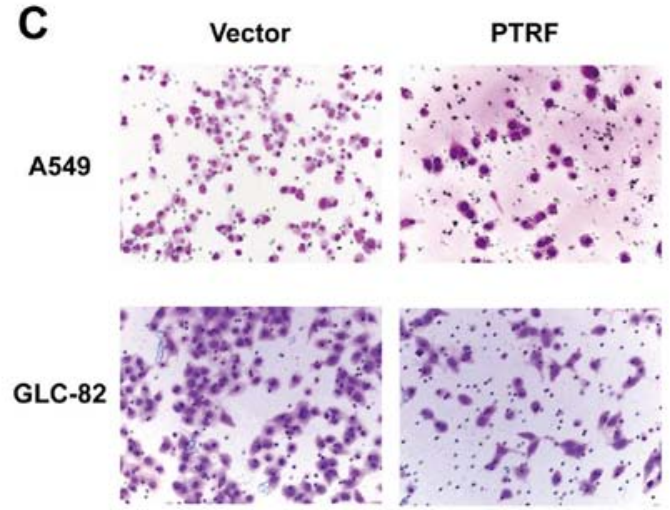

B

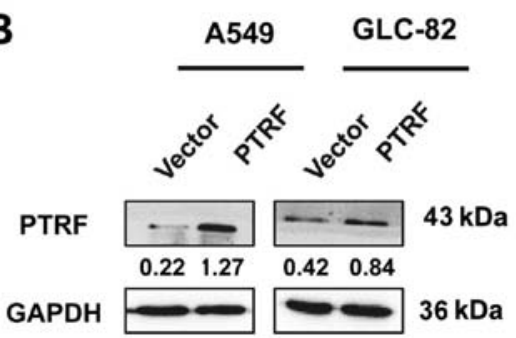

Figure 5. PTRF suppresses migration of NSCLC cells. (A) Expression of PTRF was detected in 7 NSCLC cell lines and in normal human bronchial epithelial cell line BEAS-2B using western blot analysis. (B) Western blot analysis was performed to detect PTRF in indicated cells transfected with the PTRF vector. (C) Transwell assay using control and miR-187-overexpressing or -knockdown cell lines. The cells were counted under a microscope in 5 randomly selected fields. The histograms at the right show the number of invaded cells. Representative figures are shown. The results were reproduced from 3 independent experiments. PTRF, polymerase I and transcript release factor; NSCLC, non-small cell lung cancer.

A
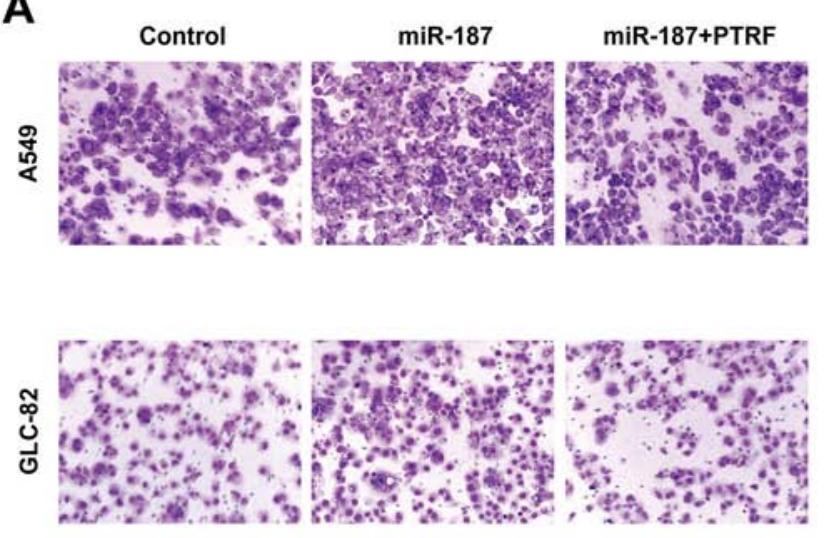
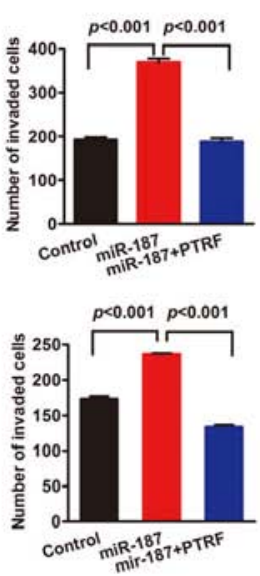

B

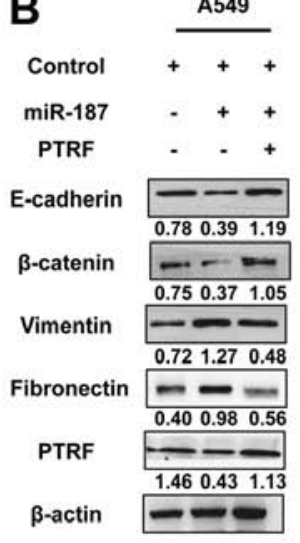

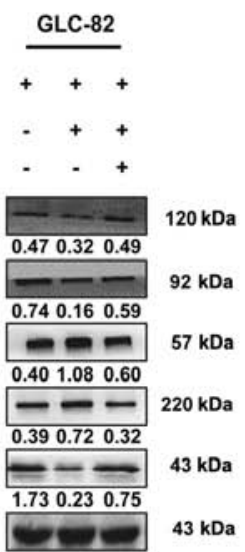

Figure 6. PTRF plays crucial roles in miR-187-induced EMT and migration of NSCLC cells. (A) Transwell assay of indicated miR-187-overexpressing cells co-transfected with PTRF. The cells were counted under a microscope in 5 randomly selected fields. The histograms at the right show the number of invaded cells. (B) Western blot analysis of EMT markers in indicated cells co-transfected with miR-187 and the PTRF vector. Representative figures are shown. The results were reproduced from 3 independent experiments. PTRF, polymerase I and transcript release factor; EMT, epithelial-mesenchynal transition; NSCLC, non-small cell lung cancer.

molecular mechanism through which miR-187 influences NSCLC progression remains largely unknown. In the present study, miR-187 was demonstrated to be a potential promoter of NSCLC progression. However, the mechanism underlying the abnormal expression of miR-187 remains unclear. The present data did not support that abnormal histone or DNA methylation was involved in abnormal expression. Our previous data revealed that miR-187 may be regulated by cell factors in colorectal cancer (CRC), such as transforming growth factor- $\beta$
(TGF $\beta$ ). Numerous recent studies have revealed that signaling pathways or non-coding RNAs also regulate miRNA expression $(25,26)$. We may investigate the regulatory mechanism of miR-187 in more detail in future studies.

The mechanism underlying the relationship between miR-187 and tumor aggressiveness remains to be elucidated. EMT is a critical step in tumor progression. Recently, the role of miRNAs in EMT has become a focus in the field of cancer research. Several studies have demonstrated that miR-200 
family members act as key regulators of EMT to enforce the epithelial phenotype (27). The suppressive role of miR-187 in ovarian cancer cell lines promoted EMT and was reported to be achieved through targeting of disabled homolog 2 (Dab2) to decrease migration and E-cadherin expression (28). However, these are currently the only observations and suggestions for the underlying molecular mechanisms. In the present study, miR-187 suppressed the epithelial phenotype and induced mesenchymal transition, strongly suggesting its critical role in the EMT process.

TGF $\beta$ is a known inducer of EMT via Smads and complementary non-Smad pathways, such as MAPK (29-31) and PI3K/AKT (32-34). In our previous study, miR-187 suppressed not only the Smad pathway but also non-Smad pathways in CRC. Restoring expression of miR-187 only partially neutralized TGF $\beta$-mediated activation of the Smad pathway by decreasing the phosphorylation level of Smad2 (20). In NSCLC, our data revealed that miR-187 could activate both the MAPK and PI3K/AKT pathways, implying that miR-187 induced NSCLC cell EMT via a non-Smad pathway, not the classical Smad pathway. Therefore, downstream targets must be explored in miR-187-induced EMT, and may be useful in designing novel specific targeted drugs for managing NSCLC metastasis. However, the specific regulator of miR-187 as a metastasis promoter is still unclear.

In general, miRNAs exert their biological function by suppressing their specific target genes at the post-transcriptional level. Recently, B7-H3 (a novel member of the B7 family) and Dab2 were reported as target genes of miR-187 in renal and ovarian cancers, respectively $(15,28)$. SOX4, NT5E and PTK6 expression was increased in CRC cells treated with TGF $\beta$, and these effects were offset by the addition of miR-187, suggesting that they are required for TGF $\beta$-induced EMT (20). However, we were not able to identify them as targets directly regulated by miR-187 in NSCLC. As previously mentioned, miR-187 may act in a cell- or organ-specific manner through the alteration of the expression of target genes, further changing their phenotypes. In the present study, all the evidence indicates that PTRF may be a direct target of miR-187. Rescued expression of PTRF restored the effect of miR-187 treatment and inactivated the downstream pathway.

PTRF, also known as cavin-1, was originally identified as a protein involved in the dissociation of transcription complexes in vitro (35). PTRF in the cell surface membrane is associated with vesicular transport, cholesterol homeostasis $(36,37)$ and lipolysis control (38). To date, the majority of studies related to PTRF have focused on prostate cancer (PC). Changes in the cell membrane involving loss of PTRF expression occur with the development of PC (39). Overexpression of PTRF in PC3 cells decreased cell motility by decreasing matrix metalloprotease 9 (MMP9) production (40). The absence of PTRF in PC cells was found to contribute significantly to tumor progression and metastasis by promoting the angiogenesis and lymphangiogenesis potential of cancer cells (41). One study supports a role for PTRF/cavin-1, through caveolae formation, as an attenuator of the non-caveolar functionality of Cav1 in Gal3-Cav1 signaling and regulation of focal adhesion dynamics and cancer cell migration (42). In addition, loss of PTRF expression has been demonstrated to be related with tumor progression in breast (43), pancreatic (44) and glioblas- toma (33) cancers. Only one publication based on proteomic assays reported PTRF/cavin-1 loss-of-expression in NSCLC tissue at the protein level, suggesting a potential role for PTRF in NSCLC development (45). Our results demonstrated for the first time that PTRF contributes to NSCLC progression through its involvement in EMT development.

In short, our results provide a basis for the concept that increased expression of miR-187 in human NSCLC may be significant in the acquisition of an aggressive phenotype. We believe that miR-187 functions as a promoter in NSCLC progression and may serve as a novel therapeutic biomarker. Moreover, the functional and/or mechanistic studies of miR-187 presented in the present study, indicate that miR-187 may play a critical role in controlling non-Smad-mediated EMT by regulating PTRF expression. This suggests that activating EMT during tumor metastasis in NSCLC may be counteracted by suppressing miR-187, a notion that can be readily tested in the clinic.

\section{Acknowledgements}

The present study was supported by the National Natural Science Foundation of China (nos. 81572813, 81272762 and 81401874), and the Guangdong Natural Science Foundation (nos. S2013010014254 and 2014A030313490).

\section{References}

1. Torre LA, Bray F, Siegel RL, Ferlay J, Lortet-Tieulent J and Jemal A: Global cancer statistics, 2012. CA Cancer J Clin 65: 87-108, 2015

2. Molina JR, Yang P, Cassivi SD, Schild SE and Adjei AA: Non-small cell lung cancer: Epidemiology, risk factors, treatment, and survivorship. Mayo Clin Proc 83: 584-594, 2008.

3. Paez JG, Jänne PA, Lee JC, Tracy S, Greulich H, Gabriel S, Herman P, Kaye FJ, Lindeman N, Boggon TJ, et al: EGFR mutations in lung cancer: Correlation with clinical response to gefitinib therapy. Science 304: 1497-1500, 2004.

4. Eccles SA and Welch DR: Metastasis: Recent discoveries and novel treatment strategies. Lancet 369: 1742-1757, 2007.

5. Kalluri R and Neilson EG: Epithelial-mesenchymal transition and its implications for fibrosis. J Clin Invest 112: 1776-1784, 2003.

6. Lin SY, Lee YX, Yu SL, Chang GC and Chen JJ: Phosphatase of regenerating liver-3 inhibits invasiveness and proliferation in non-small cell lung cancer by regulating the epithelial-mesenchymal transition. Oncotarget 7: 21799-21811, 2016.

7. Yao Y, Shi M, Liu S, Li Y, Guo K, Ci Y, Liu W and Li Y: MARVELD1 modulates cell surface morphology and suppresses epithelial-mesenchymal transition in non-small cell lung cancer. Mol Carcinog 55: 1714-1727, 2016.

8. Ambros V, Lee RC, Lavanway A, Williams PT and Jewell D: MicroRNAs and other tiny endogenous RNAs in C. elegans. Curr Biol 13: 807-818, 2003.

9. Zhu K, Ding H, Wang W, Liao Z, Fu Z, Hong Y, Zhou Y, Zhang CY and Chen X: Tumor-suppressive miR-218-5p inhibits cancer cell proliferation and migration via EGFR in non-small cell lung cancer. Oncotarget 7: 28075-28085, 2016.

10. Ma N, Zhang W, Qiao C, Luo H, Zhang X, Liu D, Zang S, Zhang L and Bai J: The tumor suppressive role of miRNA-509-5p by targeting FOXM1 in non-small cell lung cancer. Cell Physiol Biochem 38: 1435-1446, 2016.

11. Zhou L, Di Q, Sun B, Wang X, Li M and Shi J: MicroRNA-194 restrains the cell progression of non-small cell lung cancer by targeting human nuclear distribution protein C. Oncol Rep 35: 3435-3444, 2016

12. Croce CM: Causes and consequences of microRNA dysregulation in cancer. Nat Rev Genet 10: 704-714, 2009.

13. Kasinski AL and Slack FJ: Epigenetics and genetics. MicroRNAs en route to the clinic: Progress in validating and targeting microRNAs for cancer therapy. Nat Rev Cancer 11: 849-864, 2011. 
14. Chen HC, Chen GH, Chen YH, Liao WL, Liu CY, Chang KP, Chang YS and Chen SJ: MicroRNA deregulation and pathway alterations in nasopharyngeal carcinoma. Br J Cancer 100: 1002-1011, 2009.

15. Zhao J, Lei T, Xu C, Li H, Ma W, Yang Y, Fan S and Liu Y: MicroRNA-187, down-regulated in clear cell renal cell carcinoma and associated with lower survival, inhibits cell growth and migration though targeting B7-H3. Biochem Biophys Res Commun 438: 439-444, 2013.

16. Bloomston M, Frankel WL, Petrocca F, Volinia S, Alder H, Hagan JP, Liu CG, Bhatt D, Taccioli C and Croce CM: MicroRNA expression patterns to differentiate pancreatic adenocarcinoma from normal pancreas and chronic pancreatitis. JAMA 297: 1901-1908, 2007.

17. Casanova-Salas I, Rubio-Briones J, Calatrava A, Mancarella C, Masiá E, Casanova J, Fernández-Serra A, Rubio L, RamírezBackhaus M, Armiñán A, et al: Identification of miR-187 and miR-182 as biomarkers of early diagnosis and prognosis in patients with prostate cancer treated with radical prostatectomy. J Urol 192: 252-259, 2014.

18. Chen Y and Stallings RL: Differential patterns of microRNA expression in neuroblastoma are correlated with prognosis, differentiation, and apoptosis. Cancer Res 67: 976-983, 2007.

19. Sun C, Li S, Yang C, Xi Y, Wang L, Zhang F and Li D MicroRNA-187-3p mitigates non-small cell lung cancer (NSCLC) development through down-regulation of BCL6. Biochem Biophys Res Commun 471: 82-88, 2016.

20. Zhang F, Luo Y, Shao Z, Xu L, Liu X, Niu Y, Shi J, Sun X, Liu Y, Ding Y, et al: MicroRNA-187, a downstream effector of TGF $\beta$ pathway, suppresses Smad-mediated epithelial-mesenchymal transition in colorectal cancer. Cancer Lett 373: 203-213, 2016.

21. Tan X, Qin W, Zhang L, Hang J, Li B, Zhang C, Wan J, Zhou F, Shao K, Sun Y, et al: A 5-microRNA signature for lung squamous cell carcinoma diagnosis and hsa-miR-31 for prognosis. Clin Cancer Res 17: 6802-6811, 2011.

22. Wang H, An H, Wang B, Liao Q, Li W, Jin X, Cui S, Zhang Y Ding $Y$ and Zhao L: miR-133a represses tumour growth and metastasis in colorectal cancer by targeting LIM and SH3 protein 1 and inhibiting the MAPK pathway. Eur J Cancer 49: 3924-3935, 2013.

23. Cheng AM, Byrom MW, Shelton J and Ford LP: Antisense inhibition of human miRNAs and indications for an involvement of miRNA in cell growth and apoptosis. Nucleic Acids Res 33: 1290-1297, 2005.

24. Park SY, Lee JH, Ha M, Nam JW and Kim VN: miR-29 miRNAs activate p53 by targeting p85 alpha and CDC42. Nat Struct Mol Biol 16: 23-29, 2009.

25. Sun C, Li S, Zhang F, Xi Y, Wang L, Bi Y and Li D: Long non-coding RNA NEAT1 promotes non-small cell lung cancer progression through regulation of miR-377-3p-E2F3 pathway. Oncotarget 7: 51784-51814, 2016.

26. Zhang X, Wang C, Shan S, Liu X, Jiang Z and Ren T: TLR4/ROS/ miRNA-21 pathway underlies lipopolysaccharide instructed primary tumor outgrowth in lung cancer patients. Oncotarget 7: 42172-42182, 2016.

27. Gregory PA, Bracken CP, Bert AG and Goodall GJ: MicroRNAs as regulators of epithelial-mesenchymal transition. Cell Cycle 7: 3112-3118, 2008

28. Chao A, Lin CY, Lee YS, Tsai CL, Wei PC, Hsueh S, Wu TI, Tsai CN, Wang CJ, Chao AS, et al: Regulation of ovarian cancer progression by microRNA-187 through targeting Disabled homolog-2. Oncogene 31: 764-775, 2012.
29. Li NY, Weber CE, Wai PY, Cuevas BD, Zhang J, Kuo PC and Mi Z: An MAPK-dependent pathway induces epithelial-mesenchymal transition via Twist activation in human breast cancer cell lines. Surgery 154: 404-410, 2013.

30. Tomlinson DC, Baxter EW, Loadman PM, Hull MA and Knowles MA: FGFR1-induced epithelial to mesenchymal transition through MAPK/PLC $\gamma / \mathrm{COX}-2$-mediated mechanisms. PLoS One 7: e38972, 2012.

31. Gui T, Sun Y, Shimokado A and Muragaki Y: The roles of mitogen-activated protein kinase pathways in TGF- $\beta$-induced epithelial-mesenchymal transition. J Signal Transduct 2012: 289243, 2012.

32. Ning J, Liu W, Zhang J, Lang Y and Xu S: Ran GTPase induces EMT and enhances invasion in non-small cell lung cancer cells through activation of PI3K-AKT pathway. Oncol Res 21: 67-72, 2013.

33. Wang X, Liu T, Bai Y, Liao H, Qiu S, Chang Z, Liu Y, Yan X and Guo H: Polymerase I and transcript release factor acts as an essential modulator of glioblastoma chemoresistance. PLoS One 9: e93439, 2014

34. Massagué J: TGF $\beta$ signalling in context. Nat Rev Mol Cell Biol 13: 616-630, 2012.

35. Jansa P, Mason SW, Hoffmann-Rohrer U and Grummt I: Cloning and functional characterization of PTRF, a novel protein which induces dissociation of paused ternary transcription complexes. EMBO J 17: 2855-2864, 1998.

36. Cohen AW, Hnasko R, Schubert W and Lisanti MP: Role of caveolae and caveolins in health and disease. Physiol Rev 84: $1341-1379,2004$

37. Hamoudane M, Maffioli S, Cordera R, Maggi D and Salani B: Caveolin-1 and polymerase I and transcript release factor: New players in insulin-like grow th factor-I receptor signaling. J Endocrinol Invest 36: 204-208, 2013.

38. Aboulaich N, Ortegren U, Vener AV and Strålfors P: Association and insulin regulated translocation of hormone-sensitive lipase with PTRF. Biochem Biophys Res Commun 350: 657-661, 2006.

39. Gould ML, Williams G and Nicholson HD: Changes in caveolae, caveolin, and polymerase 1 and transcript release factor (PTRF) expression in prostate cancer progression. Prostate 70: 1609-1621, 2010.

40. Aung CS, Hill MM, Bastiani M, Parton RG and Parat MO: PTRF-cavin-1 expression decreases the migration of PC3 prostate cancer cells: Role of matrix metalloprotease 9. Eur J Cell Biol 90: 136-142, 2011

41. Nassar ZD, Moon H, Duong T, Neo L, Hill MM, Francois M, Parton RG and Parat MO: PTRF/Cavin-1 decreases prostate cancer angiogenesis and lymphangiogenesis. Oncotarget 4: 1844-1855, 2013

42. Meng F, Joshi B and Nabi IR: Galectin-3 overrides PTRF/Cavin-1 reduction of PC3 prostate cancer cell migration. PLoS One 10: e0126056, 2015.

43. Bai L, Deng X, Li Q, Wang M, An W, Deli A, Gao Z, Xie Y, Dai Y and Cong YS: Down-regulation of the cavin family proteins in breast cancer. J Cell Biochem 113: 322-328, 2012.

44. Liu L, Xu HX, Wang WQ, Wu CT, Chen T, Qin Y, Liu C, Xu J, Long J, Zhang B, et al: Cavin-1 is essential for the tumorpromoting effect of caveolin-1 and enhances its prognostic potency in pancreatic cancer. Oncogene 33: 2728-2736, 2014

45. Gámez-Pozo A, Sánchez-Navarro I, Calvo E, AgullóOrtuño MT, López-Vacas R, Díaz E, Camafeita E, Nistal M, Madero R, Espinosa E, et al: PTRF/cavin-1 and MIF proteins are identified as non-small cell lung cancer biomarkers by label-free proteomics. PLoS One 7: e33752, 2012. 